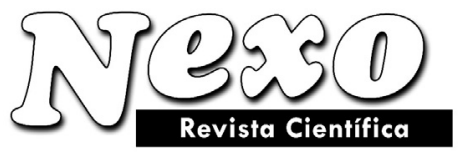

Vol. 23, No. 02, pp.62-68/Noviembre 2010

\title{
Sobre la inversión de los potenciales de Bessel-Riesz
}

\author{
R. Cerutti \\ Facultad de Ciencias Exactas y Naturales y Agrimensura \\ Universidad Nacional del Nordeste. Avda. Libertad 5540 \\ (3400) Corrientes. Argentina \\ e-mail: rcerutti@exa.unne.edu.ar
}

(recibido/received: 06-Septiembre-2010; aceptado/accepted: 12-Noviembre-2010)

\begin{abstract}
RESUMEN
En este trabajo se obtiene la inversión de un operador del tipo convolución usando técnicas de integrales hipersingulares. El operador de Bessel-Riesz de una función $\varphi$ perteneciente a $S$, el espacio de funciones de prueba de Schwartz, es definido por la convolución con las funciones generalizadas $W_{\alpha}(P \pm i 0, m, n)$ expresables en términos de la función de Bessel de primera especie $J_{\gamma} W_{\alpha}(P \pm i 0, m, n)$ es también una combinación lineal infinita del núcleo ultrahiperbólico de Riesz de diferentes ordenes. Este hecho nos permite invertir los potenciales de Bessel-Riesz de un modo análogo a lo hecho en el caso de los potenciales ultrahiperbólicos de Bessel (cf. [01]) y los potenciales causales de Riesz (cf. [2]).

Palabras Claves: Potenciales de Riesz. Integrales Hipersingulares.
\end{abstract}

\begin{abstract}
In this paper the inversion of a convolution type operator is obtained by using hypersingular integral technics. The BesselRiesz operator of a function $\varphi$ belonging to $S$, the space of test functions of Schwartz, is definied by the convolution with the generalized functions $W_{\alpha}(P \pm i 0, m, n)$ expressible in terms of the Bessel function of first kind $J_{\gamma}$. $W_{\alpha}(P \pm i 0, m, n)$ is also an infinite linear combination of the ultrahyperbolic Riesz kernel of differents orders. This fact allows us to invert the Bessel-Riesz potential in an analogue manner of the ultrahyperbolic Bessel potentials (cf. [01]) and causal Riesz potentials (cf. [2]).
\end{abstract}

Keywords: Riesz potential, hypersingular integral. 


\section{R. Cerutti}

\section{INTRODUCCIÓN}

En este artículo tratamos la inversión de ciertos operadores de tipo potencial notados $W^{\alpha} \varphi$ que son convoluciones con una función distribucional perteneciente a una familia de funciones dependientes de un parámetro complejo $\alpha$, $\left\{W_{\alpha}(P \pm i 0, m, n)\right\}_{\alpha \in \mathbb{C}}$ y $\varphi$ una función del espacio $S$ de funciones de Schwartz.

Trione (cf. [3]) introdujo la familia $\left\{W_{\alpha}(P \pm i 0, m, n)\right\}_{\alpha \in \mathbb{C}}$ mediante la expresión

$$
W_{\alpha}(P \pm i 0, m, n)=\frac{\left[m^{-1}(P \pm i 0)^{\frac{1}{2}}\right]^{\frac{\alpha-n}{2}}}{(-1) \pi^{\frac{n-2}{2}} 2^{\frac{n+\alpha}{2}} \Gamma\left(\frac{\alpha}{2}\right)} J_{\frac{\alpha-n}{2}}\left[m(P \pm i 0)^{\frac{1}{2}}\right]
$$

donde $m$ es un numero real no negativo, y $J_{\gamma}$ es la función de Bessel de primera especie.

Conociendo la transformada de Fourier de la función generalizada $(P \pm i 0)^{\lambda}$ (cf. [10]) puede calcularse la transformada de Fourier de $W_{\alpha}(P \pm i 0, m, n)$. Trione obtuvo que (cf. [4])

$$
\mathfrak{F}\left[W_{\alpha}(P \pm i 0, m, n)\right]=\sum_{\gamma=0}^{\infty}\left(\begin{array}{c}
-\frac{\alpha}{2} \\
\gamma
\end{array}\right) m^{2 \gamma}(Q \mp i 0)^{-\frac{\alpha+2 \gamma}{2}}
$$

Sea $\varphi$ una función perteneciente a $S$, el espacio de Schwartz de funciones decrecientes en infinito más rápido que $|x|^{-1}$. El potencial de Bessel-Riesz de orden $\alpha$ esta dado, por definición, por la siguiente convolución

$$
W^{\alpha} \varphi=W_{\alpha}(P \pm i 0, m, n) * \varphi
$$

Usando una expresión equivalente de la función generalizada $(P \pm i 0)$ en términos de las funciones generalizadas $P_{+} \mathrm{y}$ $P_{-}$que se definen de la siguiente manera:

$$
P_{+}^{\lambda}=\left\{\begin{array}{ll}
P^{\lambda} & \text { if } P>0 \\
0 & \text { if } P<0
\end{array} ; \quad P_{-}^{\lambda}= \begin{cases}0 & \text { if } P>0 \\
|P|^{\lambda} & \text { if } P<0\end{cases}\right.
$$

De (cf. [4]) resulta que

$$
(P \pm i 0)^{\lambda}=P_{+}^{\lambda} \pm e^{i \pi \lambda} P_{-}^{\lambda}
$$

Teniendo en cuenta esta última expresión y que la función $W_{\alpha}(P \pm i 0, m, n)$ puede expresarse como una combinación lineal infinita de las funciones generalizadas $(P \pm i 0)$ (cf. [3]), la convolución en (3) tiene la forma

$$
W^{\alpha} \varphi=\sum_{\gamma=0}^{\infty}\left(\begin{array}{c}
-\frac{\alpha}{2} \\
\gamma
\end{array}\right) m^{2 \gamma}\left[\int_{K_{+}} P^{\frac{\alpha-n+2 \gamma}{2}} \varphi(x-t) d t+e^{i \pi \frac{(\alpha-n+2 \gamma)}{2}} \int_{K_{-}}|P|^{\frac{\alpha-n+2 \gamma}{2}} \varphi(x-t) d t\right]
$$

donde $K_{+}$y $K_{-}$denotan los conos: $K_{+}=\left\{t \in \mathbb{R}^{n}: P(t)>0\right\}, K_{-}=\left\{t \in \mathbb{R}^{n}: P(t)<0\right\}$. 


\section{R. Cerutti}

La integral en (3) converge si $\alpha+2 \gamma>n-2$ y en el caso $\alpha+2 \gamma \leq n-2$ admite una prolongación analítica respecto de $\alpha$.

\section{Inversion de los potenciales de bessel-riesz}

Siguiendo a Rubin (cf. [5]) el problema de la inversión es equivalente en términos de la transformada de Fourier a la división por cierta potencia de la función generalizada $(P \pm i 0)$.

Sea $\alpha$ un numero real no negativo, $\alpha<l ; l>0$; para cada entero no negativo $\gamma$, tal que $\alpha-2 \gamma>0$, sea $T_{l, \varepsilon, \gamma}^{\alpha-2 \gamma} f$ el siguiente operador

$$
\left(T_{l, \varepsilon, \gamma}^{\alpha-2 \gamma} f\right)(x)=\int_{\mathbb{R}^{n}}\left(P+i \varepsilon|t|^{2}\right)^{-\frac{n+\alpha-2 \gamma}{2}}\left\{\left(\Delta_{t}^{l} f\right)(x)\right\} d t
$$

donde $\left(\Delta_{t}^{l} f\right)(x)=\sum_{k=0}^{\infty}\left(\begin{array}{l}l \\ k\end{array}\right)(-1)^{k} f(x-k t)$ es la diferencia de orden $l$ de la función $f$ en el punto $x$ con intervalo $t$. El operador $T_{l, \varepsilon, \gamma}^{\alpha-2 \gamma} f$ se define como "integral hipersingular en diferencias" y es el análogo causal de la integral definida por Samko (cf. [6]) para el caso elíptico y de la integral definida por Rubin (cf. [7]) para el caso de los potenciales de Bessel y por nosotros (cf. [1]) para el caso de los potenciales causales de Bessel y para los potenciales causales de Riesz (cf. [2], y [8]).

Puede observarse que el operador $T_{l, \varepsilon, \gamma}^{\alpha-2 \gamma} f$ depende de la función generalizada $\left(P+i \varepsilon|t|^{2}\right)$, que es una forma cuadrática con coeficientes complejos cuya parte imaginaria es positiva. Luego, análogamente a lo hecho por Gelfand (cf. [4]) y Trione (cf. [9]) evaluaremos su transformada de Fourier como si él dependiera de $|t|^{2}$, es decir como si fuera una distribución radial invariante por rotaciones, y finalmente en el resultado haremos la sustitución de $|\xi|^{2}$ por $\left(Q-i \varepsilon|\xi|^{2}\right)$.

Sea $\mathfrak{F}\left[T_{l, \varepsilon, \gamma}^{\alpha-2 \gamma} f\right](\xi)$ la transformada de Fourier de (7), y sea $P$ una forma cuadrática definida positiva, entonces

$$
\begin{aligned}
\mathfrak{F}\left[T_{l, \varepsilon, \gamma}^{\alpha-2 \gamma} f\right](\xi) & =\mathfrak{F}\left[\int_{\mathbb{R}^{n}}\left(P+i \varepsilon|t|^{2}\right)^{-\frac{n+\alpha-2 \gamma}{2}}\left\{\left(\Delta_{t}^{l} f\right)(x)\right\} d t\right]= \\
& =\mathfrak{F}\left[\int_{\mathbb{R}^{n}}\left(|t|^{2}\right)^{-\frac{n+\alpha-2 \gamma}{2}}\left\{\left(\Delta_{t}^{l} f\right)(x)\right\} d t\right]_{|\xi|^{2} \rightarrow Q \mp i \varepsilon|\xi|^{2}}
\end{aligned}
$$

donde el símbolo que aparece en el segundo miembro es el mismo que aparece en el Teorema debido a Trione (cf. [9]). Luego se tiene que

$$
\begin{aligned}
\mathfrak{F}\left[T_{l, \varepsilon, \gamma}^{\alpha-2 \gamma} f\right](\xi) & =\sum_{k=0}^{l}\left(\begin{array}{l}
l \\
k
\end{array}\right)(-1)^{k} \int_{\mathbb{R}^{n}} \mid t^{-(n+\alpha-2 \gamma)} d t \int_{\mathbb{R}^{n}} e^{i\langle x, \xi\rangle} f(x-k t) d x= \\
& =\sum_{k=0}^{l}\left(\begin{array}{l}
l \\
k
\end{array}\right)(-1)^{k} \mathfrak{F}[f](\xi) \int_{\mathbb{R}^{n}} e^{i k\langle t, \xi\rangle}|t|^{-(n+\alpha-2 \gamma)} d t
\end{aligned}
$$




\section{R. Cerutti}

Haciendo el cambio de variables $k t=y$, y aplicando la formula (4) página 263 de [4] se obtiene

$$
\mathfrak{F}\left[T_{l, \varepsilon, \gamma}^{\alpha-2 \gamma} f\right](\xi)=\mathfrak{F}[f](\xi) \sum_{k=0}^{l}\left(\begin{array}{l}
l \\
k
\end{array}\right)(-1)^{k} k^{\alpha-2 \gamma} \pi^{\frac{n}{2}} \frac{\Gamma\left(-\frac{\alpha-2 \gamma}{2}\right)}{\Gamma\left(\frac{n+\alpha-2 \gamma}{2}\right)}|\xi|^{\alpha-2 \gamma}
$$

y realizando la sustitución $|\xi|^{2}$ by $Q \mp i \varepsilon|\xi|^{2}$, resulta que

$$
\mathfrak{F}\left[T_{l, \varepsilon, \gamma}^{\alpha-2 \gamma} f\right](\xi)=\frac{\mathbf{A}_{l}(\alpha-2 \gamma)}{2^{\alpha} \Gamma\left(\frac{n+\alpha-2 \gamma}{2}\right)} e^{i \frac{\pi}{2} q} \pi^{\frac{n}{2}} \Gamma\left(-\frac{\alpha-2 \gamma}{2}\right) \mathfrak{F}[f](\xi)\left(Q \mp i \varepsilon|\xi|^{2}\right)^{\frac{\alpha-2 \gamma}{2}}
$$

válida para $\alpha \neq 2,4, \ldots$, donde

$$
\mathbf{A}_{l}(\alpha-2 \gamma)=\sum_{k=0}^{l}\left(\begin{array}{l}
l \\
k
\end{array}\right)(-1)^{k} k^{\alpha-2 \gamma}
$$

Usando las conocidas relaciones $z \Gamma(z) \Gamma(-z)=\Gamma(z) \Gamma(1-z) \mathrm{y} ; \Gamma\left(-\frac{\alpha}{2}\right)=\frac{\pi}{\Gamma\left(1+\frac{\alpha}{2}\right) \operatorname{sen} \frac{\pi \alpha}{2}}$ la formula puede escribirse, para $\alpha \neq 2,4, \ldots$, como sigue

$$
\mathfrak{F}\left[T_{l, \varepsilon, \gamma}^{\alpha-2 \gamma} f\right](\xi)=\frac{\pi^{\frac{n}{2}+1} e^{i \frac{\pi}{2} q} \mathbf{A}_{l}(\alpha-2 \gamma)}{2^{\alpha-2 \gamma} \Gamma\left(1+\frac{\alpha-2 \gamma}{2}\right) \Gamma\left(\frac{n+\alpha-2 \gamma}{2}\right) \operatorname{sen} \frac{\pi}{2}(\alpha-2 \gamma)}\left(Q \mp i \varepsilon|\xi|^{2}\right)^{\frac{\alpha-2 \gamma}{2}} \mathfrak{F}[f](\xi)
$$

Cuando $\alpha-2 \gamma=2,4, \ldots$ se tiene

$$
\mathfrak{F}\left[T_{l, \varepsilon, \gamma}^{\alpha-2 \gamma} f\right](\xi)=\frac{(-1)^{\alpha-2 \gamma} \pi^{\frac{n}{2}} 2^{1-(\alpha-2 \gamma)} e^{i \frac{\pi}{2} q}}{\Gamma\left(1+\frac{\alpha-2 \gamma}{2}\right) \Gamma\left(\frac{n+\alpha-2 \gamma}{2}\right)} \frac{d}{d \alpha} \mathbf{A}_{l}(\alpha-2 \gamma)\left(Q \mp i \varepsilon|\xi|^{2}\right)^{\frac{\alpha-2 \gamma}{2}} \mathfrak{F}[f](\xi)
$$

De (13) y (14) se concluye que

$$
\mathfrak{F}\left[T_{l, \varepsilon, \gamma}^{\alpha-2 \gamma} f\right](\xi)=d_{n, l}(\alpha-2 \gamma)\left(Q \mp i \varepsilon|\xi|^{2}\right)^{\frac{\alpha-2 \gamma}{2}} \mathfrak{F}[f](\xi)
$$

donde $d_{n, l}(\alpha-2 \gamma)$ es análoga a las constantes que aparecen en el caso de los potenciales de Riesz elípticos y de los potenciales de Bessel y causales de Bessel (cf. [7], [6], [1], [8]).

De las anteriores consideraciones resulta valido el siguiente

Teorema 1: Sea $\alpha$ un numero real, $\alpha \neq-\frac{n}{2}-k, k=0,1,2, \ldots, \alpha<l ; l$ un entero no negativo. Sea $f$ una función perteneciente a $S$, y sea $\gamma$ un entero no negativo fijo tal que $\alpha-2 \gamma>0$.

Entonces la integral hipersingular de orden $\alpha-2 \gamma ; T_{l, \varepsilon, \gamma}^{\alpha-2 \gamma} f$ existe y su transformada de Fourier es

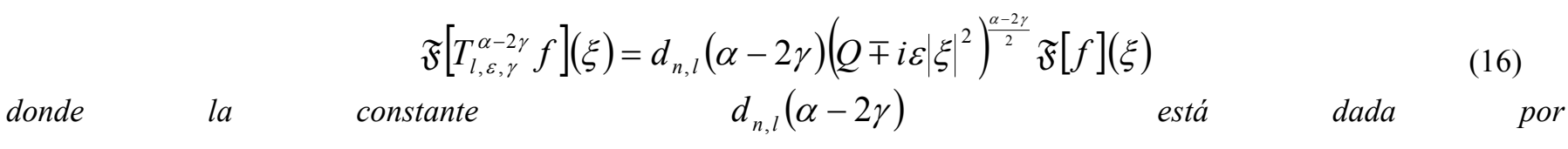




$$
d_{n, l}(\alpha-2 \gamma)=\left\{\begin{array}{lc}
\frac{\pi^{\frac{n}{2}+1} e^{i \frac{\pi}{2} q} \mathbf{A}_{l}(\alpha-2 \gamma)}{2^{\alpha-2 \gamma} \Gamma\left(1+\frac{\alpha-2 \gamma}{2}\right) \Gamma\left(\frac{n+\alpha-2 \gamma}{2}\right) \operatorname{sen} \frac{\pi}{2}(\alpha-2 \gamma)} & \text { If } \alpha \neq 2,4,6 \ldots \\
\frac{(-1)^{\alpha-2 \gamma} \pi^{\frac{n}{2}} 2^{1-(\alpha-2 \gamma)} e^{i \frac{\pi}{2} q} \frac{d}{\Gamma\left(1+\frac{\alpha-2 \gamma}{2}\right) \Gamma\left(\frac{n+\alpha-2 \gamma}{2}\right)} \frac{\mathbf{A}_{l}(\alpha-2 \gamma)}{d \alpha}}{\text { If } \alpha=2,4,6 \ldots} &
\end{array}\right.
$$

\section{La derivada generalizada de bessel-riesz}

El propósito en esta sección es obtener un operador inverso de $W^{\alpha}$, que será indicado por $\left(W^{\alpha}\right)^{-1}$, tal que si $f=W^{\alpha} \varphi$ resulte que $\varphi=\left(W^{\alpha}\right)^{-1} f$. Para hacer eso se introduce el siguiente operador que notaremos $\left(W^{\alpha}\right)^{-1}$ definiéndolo del siguiente modo

$$
\left(W^{\alpha}\right)^{-1}=\sum_{\gamma=0}^{\left[\frac{\alpha}{2}\right]}\left(\begin{array}{c}
\frac{\alpha}{2} \\
\gamma
\end{array}\right) \frac{m^{2 \gamma} T_{l}^{\alpha-2 \gamma} f}{d_{n, l}(\alpha-2 \gamma)}+\sum_{\gamma \geq\left[\frac{\alpha}{2}\right]+1}^{\infty}\left(\begin{array}{c}
\frac{\alpha}{2} \\
\gamma
\end{array}\right) m^{2 \gamma} \frac{R_{-\alpha+2 \gamma}}{H(-\alpha-2 \gamma)} * f
$$

Donde

$$
T_{l}^{\alpha-2 \gamma} f=\lim _{\varepsilon \rightarrow 0} T_{l, \varepsilon}^{\alpha-2 \gamma} f
$$

$\left(W^{\alpha}\right)^{-1}$ es un operador que es combinación lineal de derivadas causales de Riesz de orden $\alpha-2 \gamma, \gamma=0,1, \cdots,\left[\frac{\alpha}{2}\right]$ más un operador integral, cuya transformada de Fourier esta dada por

$$
\mathfrak{F}\left[\left(W^{\alpha}\right)^{-1}(f)\right](\xi)=\sum_{\gamma=0}^{\left[\frac{\alpha}{2}\right]}\left(\begin{array}{c}
\frac{\alpha}{2} \\
\gamma
\end{array}\right) m^{2 \gamma}(Q-i 0)^{\frac{\alpha-2 \gamma}{2}} \mathfrak{F}[f]+\sum_{\gamma \geq\left[\frac{\alpha}{2}\right]+1}^{\infty}\left(\begin{array}{c}
\frac{\alpha}{2} \\
\gamma
\end{array}\right) m^{2 \gamma}(Q-i 0)^{\frac{\alpha}{2}-\gamma} \mathfrak{F}[f]
$$

Observando que cada término del segundo miembro en tiene la misma forma, puede escribirse

$$
\mathfrak{F}\left[\left(W^{\alpha}\right)^{-1}(f)\right](\xi)=\sum_{\gamma \geq 0}\left(\begin{array}{c}
\frac{\alpha}{2} \\
\gamma
\end{array}\right) m^{2 \gamma}(Q-i 0)^{\frac{\alpha-2 \gamma}{2}} \mathfrak{F}[f](\xi)
$$

y teniendo en cuenta que

$$
\mathfrak{F}\left[W_{\alpha}(P \pm i 0, m, n)\right]=\sum_{\gamma=0}^{\infty}\left(\begin{array}{c}
-\frac{\alpha}{2} \\
\gamma
\end{array}\right) m^{2 \gamma}(Q \mp i 0)^{-\frac{\alpha+2 \gamma}{2}}
$$

resulta

$$
\mathfrak{F}\left[W_{-\alpha} * f\right]=\sum_{\gamma=0}^{\infty}\left(\begin{array}{c}
\frac{\alpha}{2} \\
\gamma
\end{array}\right) m^{2 \gamma}(Q-i 0)^{\frac{\alpha}{2}-\gamma}=\mathfrak{F}\left[\left(W^{\alpha}\right)^{-1}(f)\right]
$$

De modo análogo a lo hecho para definir la derivada de Riesz (cf. [6]), la derivada causal de Riesz (cf. [8]) y la derivada 
causal de Bessel (cf. [1]) definimos la derivada generalizada de Bessel-Riesz de orden $\alpha$ de una función $f$ perteneciente a $S$ para $\alpha \neq 1,3,5, \ldots \mathrm{D}^{\alpha}$ como el operador, que expresado mediante su transformada de Fourier, esta dado por la siguiente expresión:

$$
\mathfrak{F}\left[\mathbf{D}^{\alpha} f\right](\xi)=\sum_{\gamma=0}^{\infty}\left(\begin{array}{c}
\frac{\alpha}{2} \\
\gamma
\end{array}\right) m^{2 \gamma}(Q \mp i 0)^{\frac{\alpha-2 \gamma}{2}} \mathfrak{F}[f](\xi)
$$

En lo que sigue probaremos que $\mathrm{D}^{\alpha}$ es un operador inverso a izquierda del operador $W^{\alpha}$.

Teorema 2 Sea $\varphi$ una función de $S, \alpha$ un número real positivo, $\alpha \neq 1,3,5, \ldots \quad \frac{n+\alpha}{2} \neq-\frac{n}{2}-k ; k=0,1,2, \ldots y$ $\mathbf{D}^{\alpha} \varphi=f$, para $f \in S$. Entonces $\varphi=W^{\alpha} f$.

Demostración: Como por hipotesis $\mathrm{D}^{\alpha} \varphi=f$ aplicando transformada de Fourier a ambos miembros se tiene que $\mathfrak{F}\left[\mathbf{D}^{\alpha} \varphi\right]=\mathfrak{F}[f]$.

Considerando (24), puede escribirse

$$
\mathfrak{F}[f]=\sum_{\gamma=0}^{\infty}\left(\begin{array}{c}
\frac{\alpha}{2} \\
\gamma
\end{array}\right) m^{2 \gamma}(Q \mp i 0)^{\frac{\alpha+2 \gamma}{2}} \mathfrak{F}[\varphi](\xi)
$$

y teniendo en cuenta (23), puede escribirse

$$
\mathfrak{F}[f]=\mathfrak{F}\left[W_{-\alpha}(P \pm i 0, m, n) * \varphi\right]
$$

De las propiedades del núcleo $W_{\alpha}(P \pm i 0, m, n)$ y por la unicidad de la transformada de Fourier resulta $W_{\alpha}(P \pm i 0, m, n) * f=\varphi, \quad$ o equivalentemente

$$
W^{\alpha} f=\varphi
$$

que es la tesis del Teorema 2.

\section{REFERENCIAS}

[1] Cerutti, R.A. The ultrahyperbolic Bessel operator: an inversion theorem. Mathematical and Computer Modelling. Vol. 22, No2. 1995

[2] Cerutti, R.A. On the inversion of causal Riesz potentials. Trabajos de Matemática №248, Instituto Argentino de Matemática, CONICET - UBA. 1995

[3] Trione, S.E. On elementary $(P \pm i 0)^{\lambda} \quad$ ultrahyperbolic solution of the Klein-Gordon operator iterated $k$ times. Integral Transforms and Special Functions. Vol. 9. No 2; pp. 149-162. 2000.

[4] Gelfand,I.M. and Shilov, G.E., Generalized Functions, Vol I, Academic Press, New York, 1964.

[5] Rubin, B. Fractional integral and potentials. Pitman Monograhs 82. Longman. 1996.

Vol. 23, No. 02, pp. 62-68/Noviembre 2010 


\section{R. Cerutti}

[6] Samko, S.G. On spaces of Riesz potentials. Math. USSR, Izvestiya, Vol. 10, No 5, pp. 1089-1117. 1976.

[7] Rubin, B. Description and inversion of Bessel potentials by using hypersingular integrals with weighted differences. Differential Equations, 22. Nº 10, pp. 1246-1256. 1987.

[8] Cerutti, R.A. and Trione, S.E. Some properties of the generalized causal and anticausal Riesz potentials. Applied Math Letters, 13 2000, 129-136.

[9] Trione, S.E., Distributional products, Cursos de Matemática, № 3, Instituto Argentino de Matemática, IAMCONICET, Buenos Aires, Argentina, 1980.

[10] Riesz, M. L'integral de Riemann-Liouville et le problème de Cauchy, Acta Mathematica, 81, pp. 1-223.1949.

[11] Trione, S.E., Sobre núcleos ultrahiperbólicos de Marcel Riesz y sus propiedades. Anales de la Academia Nacional de Ciencias de Buenos Aires. 1997.

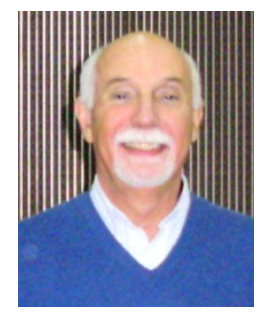

Ruben Alejandro Cerutti: Doctor en Matematica por la Universidad Nacional del Nordeste, UNNE, Argentina y diplomado en Historia de las Ciencias por la Universidad de Zaragoza, España.

Profesor Titular de Analisis de variable compleja en la Facultad de Ciencias Exactas de la UNNE y de Analisis Matematico del Profesorado en Matematica de la Universidad Nacional de Formosa,Argentina. 